Pure Appl. Chem., Vol. 76, No. 5, pp. 907-914, 2004.

(C) 2004 IUPAC

\title{
Predicting drug metabolism: Concepts and challenges*
}

\author{
Bernard Testa ${ }^{1, \ddagger}$, Anne-Loyse Balmat ${ }^{1}$, and Anthony Long ${ }^{2}$ \\ ${ }^{1}$ Institute of Medicinal Chemistry, University of Lausanne, CH-1015 Lausanne, \\ Switzerland; ${ }^{2}$ Lhasa Ltd., Department of Chemistry, University of Leeds, \\ Woodhouse Lane, Leeds LS2 9JT, UK
}

\begin{abstract}
The paper begins with a discussion of the needs and goals of metabolic predictions in early drug research. Major difficulties toward this objective are examined, mainly the various substrate and product selectivities characteristic of drug metabolism. In a second part, we classify and summarize the major in silico methods used to predict drug metabolism. A discrimination is thus made between "local" and "global" systems. In the last part of the paper, the program METEOR is presented and evaluated using the published metabolic data of 10 substrates.
\end{abstract}

\section{DRUG METABOLISM AND THE MEDICINAL CHEMIST}

\section{Why are medicinal chemists concerned with drug metabolism?}

Current "drug" discovery is mainly a ligand design aimed at discovering compounds with high affinity/activity toward predefined biological targets. Modern high-throughput techniques have rendered this strategy immensely successful, but much remains to be done to transform hits and ligands into well-behaved leads and clinical candidates. To decrease the costly and time-consuming development of active compounds ultimately doomed by hidden pharmacokinetic or toxicological defects, medicinal chemists now integrate metabolic considerations into drug design and lead optimization strategies [1]. As a result, many aspects of drug metabolism are of interest to medicinal chemists, including [2]:

- $\quad$ the chemistry and biochemistry of metabolic reactions;

- the consequences of such reactions on activation and inactivation, toxification and detoxification;

- $\quad$ predictions of drug metabolism based on quantitative structure-metabolism relationships, expert systems, and molecular modeling of enzymatic sites;

- $\quad$ prodrug and soft drug design; and

- $\quad$ changes in physicochemical properties (acidity, basicity, lipophilicity, etc.) resulting from biotransformation.

Major reasons for the ever-increasing significance of biotransformation in drug discovery and development are its frequent pharmacodynamic and pharmacokinetic consequences [2-4], as resulting from the formation of:

- $\quad$ active metabolites from active drugs;

- $\quad$ active metabolites from prodrugs (activation);

- $\quad$ inactive metabolites (inactivation);

*Lecture presented at the Polish-Austrian-German-Hungarian-Italian Joint Meeting on Medicinal Chemistry, Kraków, Poland, 15-18 October 2003. Other presentations are published in this issue, pp. 907-1032.

${ }^{\ddagger}$ Corresponding author: Present address: Pharmacy Dept, University Hospital, Rue du Bugnon, CH-1011 Lausanne-CHUV, Switzerland; E-mail: Bernard.Testa@hospvd.ch. 
- toxic metabolites (toxification), and particularly reactive metabolites able to form covalent adducts with critical biomolecules;

- $\quad$ metabolites capable of inhibiting a metabolic pathway, producing complex kinetics; and

- metabolites having physicochemical properties vastly different from those of the parent compound, for example, a very high lipophilicity resulting in tissue accumulation and residue retention.

These reasons have created a strong incentive for medicinal chemists to be able to anticipate the biotransformation of any given compound, including the generation of reactive metabolites [5]. Whereas human expertise is irreplaceable, there is now a clear need for expert systems aimed at providing reliable and versatile metabolic predictions.

\section{Goals of metabolic prediction}

"Metabolic prediction" in itself is a fuzzy and broad concept which calls for definition and clarification. A number of stepwise goals toward predicting the metabolism of a given compound are listed here [2]:

Goal 1. A list of all reasonable phase I and phase II metabolites

Goal 2. Same as above, organized in a metabolic tree

Goal 3. Same as above, plus a warning for reactive/adduct-forming metabolites

Goal 4. Same as above, plus (a) a probability of formation based on molecular factors, and (b) a filter against improbable metabolites

Goal 5. Same as above, plus a probability of formation under different biological conditions

Predicting all reasonable metabolites (Goal 1) represents the simplest goal. For want of a better definition, we take "a reasonable metabolite" to be one that can be postulated on the grounds that the substructural motif (functional group) undergoing the biotransformation has a precedent in the metabolic chemistry literature, i.e., one not postulated solely on a mechanistic hypothesis. Classifying the predicted metabolites into a metabolic tree (Goal 2) calls for additional rules, as does the identification of potentially reactive and/or adduct-forming metabolites or metabolic intermediates (Goal 3). The difficulty reaches new heights when semi-quantitative predictions are sought, based on molecular properties of the substrate (Goal 4). The necessary rules must also originate in existing knowledge, but they should be derived from structure-metabolism relationships using such statistical tools as multivariate analyses and neural networks. The same is true for the highest level of difficulty, when biological factors are taken into account to modulate the predictions according to animal species, genetic factors, age, etc. (Goal 5). In other words, the goal of the ultimate expert system would be to generate condition-dependent, semi-quantitative metabolic trees, a goal that will only be met very progressively.

\section{Challenge of substrate and product selectivities}

There are a number of challenges to reliable drug metabolism prediction, foremost among which are the many biological factors [2]:

- $\quad$ interindividual factors (i.e., which remain invariable for a given organism): animal species, genetic factors, gender;

- $\quad$ intraindividual factors (i.e., which vary for a given organism): age, biological rhythms, disease, stress, pregnancy, nutrition, influence of inducers and inhibitors.

Another major challenge in reliable prediction of metabolism, and one which deserves more interest than it receives, arises from the various selectivities characteristic of metabolic processes. Whereas there is only one type of selectivity at the receptor level, namely, the quantitatively or qualita- 
tively different responses elicited by various pharmacodynamic agents, two different types of selectivity exist in xenobiotic metabolism, namely, substrate selectivity and product selectivity (Table 1) $[2,3]$.

Table 1 Types of selectivity seen in xenobiotic metabolism.

\begin{tabular}{|c|c|}
\hline $\begin{array}{l}\text { Substrate selectivity (broad sense) } \\
\text { Distinct substrates are metabolized: } \\
\text { - at different rates } \\
\text { - under identical conditions }\end{array}$ & $\begin{array}{l}\text { Product selectivity } \\
\text { Distinct metabolites are produced: } \\
\text { - from a single substrate } \\
\text { - at different rates } \\
\text { - under identical conditions }\end{array}$ \\
\hline $\begin{array}{l}\text { Substrate selectivity (narrow sense) } \\
\text { (nonisomeric substrates) }\end{array}$ & $\begin{array}{l}\text { Chemoselectivity } \\
\text { (chemically distinct reactions) }\end{array}$ \\
\hline $\begin{array}{l}\text { Substrate regioselectivity } \\
\text { (regioisomeric substrates) }\end{array}$ & $\begin{array}{l}\text { Product regioselectivity } \\
\text { (regioisomeric products) }\end{array}$ \\
\hline $\begin{array}{l}\text { Substrate stereoselectivity } \\
\text { (stereoisomeric substrates) }\end{array}$ & $\begin{array}{l}\text { Product stereoselectivity } \\
\text { (stereoisomeric products) }\end{array}$ \\
\hline
\end{tabular}

Substrate-product selectivities

Substrate selectivity is defined as the differential metabolism of distinct substrates under identical conditions; its analogy with pharmacodynamic processes is clear. In contrast, product selectivity (defined as the differential formation of distinct metabolites from a single substrate under identical conditions) has no known correspondence in receptor-mediated events. Both types of selectivity can be subdivided into subtypes depending whether substrates (or products) are nonisomeric (e.g., homologs, analogs, or congeners), regioisomeric (i.e., positional isomers), or stereoisomeric (diastereomers or enantiomers). These subtypes are listed and defined in Table 1 . Product selectivity may also vary among substrates, i.e., it may be substrate-selective (substrate-product selectivity).

Both substrate and product selectivity are of utmost significance when attempting to predict biotransformation. Indeed, substrate selectivity offers the conceptual framework to rank substrates according to their relative rate of biotransformation by a given enzyme, in a given reaction, in a given organ, in a given organism, etc. Product selectivity is even more important when a single substrate is considered, since it allows us to make sense of the relative rates of formation of metabolites generated by different routes, different enzymes, or even resulting from attack by the same enzyme at different positions in the molecule. Thus, chemoselectivity will be observed when different types of atoms are attacked (e.g., of $O$ - vs. $N$-glucuronidation, $N$ - vs. $S$-oxygenation, $\mathrm{Csp}^{3}$ vs. $\mathrm{Csp}^{2}$ hydroxylation), whereas regioselectivity implies that the same type of atom exists in the two or more positions being attacked (e.g., ortho- vs. para-hydroxylation). There is even some overlap between chemoselectivity and regioselectivity, e.g., phenol vs. alcohol glucuronidation, the chemical difference between the ether glucuronides so formed being small.

\section{IN SILICO SYSTEMS TO PREDICT METABOLISM}

In a schematic manner (Table 2), one can distinguish between two types of algorithms to predict drug and xenobiotic metabolism [2], namely, "local" systems and "global" systems.

\section{“Local” systems}

"Local" systems apply to single enzymes or to single metabolic reactions, and they are usually restricted to rather narrow chemical series. Such systems include quantitative structure-metabolism relationships (QSMRs) based on structural and physicochemical properties [6]. Quantum mechanical calculations 
may also shed light on SMRs, revealing correlations between rates of metabolic oxidation and energy barrier in cleavage of the target $\mathrm{C}-\mathrm{H}$ bond [7]. Three-dimensional QSMRs (3D-QSMRs) methods yield a partial view of the binding/catalytic site of a given enzyme as derived from the 3D molecular fields of a series of substrates or inhibitors (the training set). In other words, they yield a "photographic negative" of such sites, and will allow a quantitative prediction for novel compounds structurally related to the training set [8]. Two popular methods in 3D-QSARs are CoMFA (comparative molecular field analysis) and Catalyst.

Table 2 A classification of in silico methods to predict biotransformation.

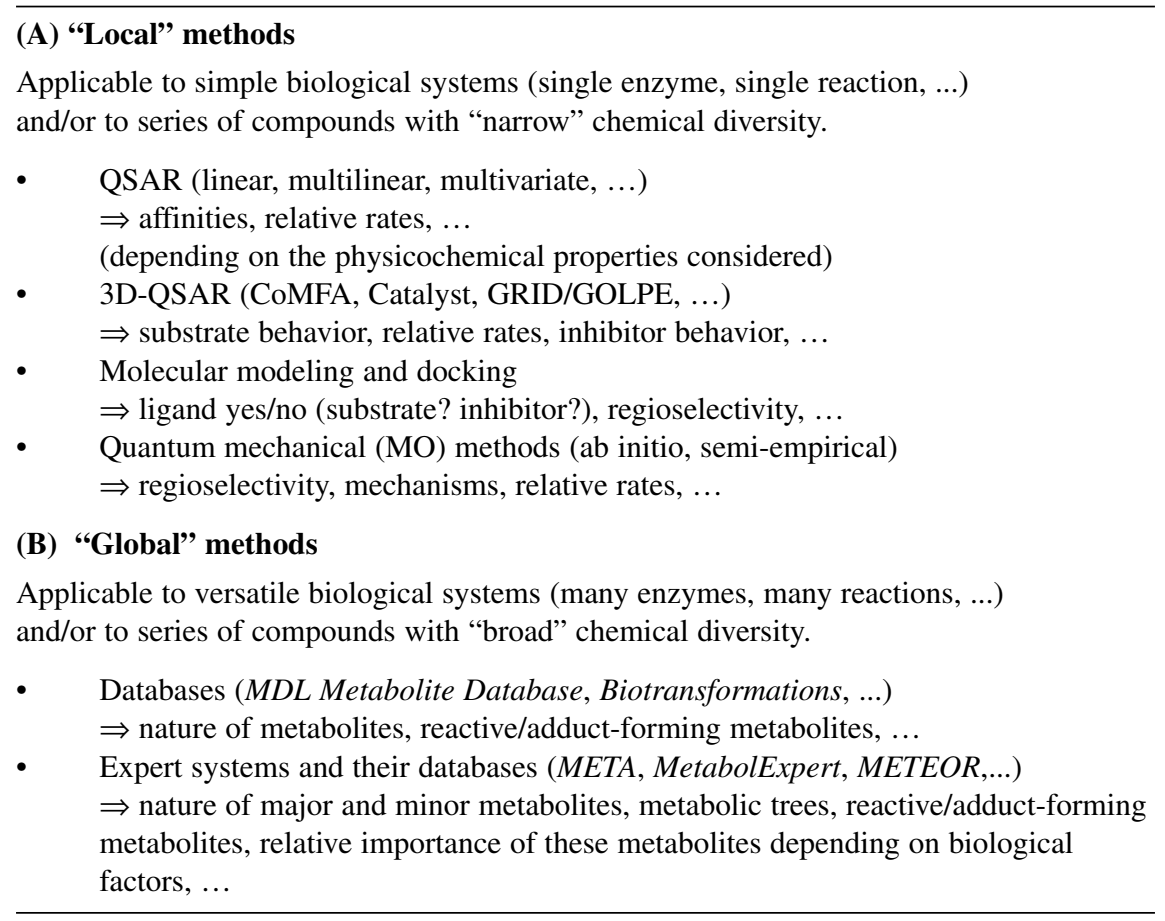

The molecular modeling of xenobiotic-metabolizing enzymes affords another approach to rationalize and predict drug-enzyme interactions [9]. Its application to drug metabolism was made possible by the crystallization and X-ray structural determination of cytochromes P450. Given the assumptions made in homology modeling and the lack of accurate scoring functions, such pharmacophoric models cannot give quantitative affinity predictions. However, they can afford fairly reliable yes/no answers as to the affinity of test set compounds, and even the regioselectivity of metabolic attack [10].

The pharmacophoric models of a large number of mammalian and mostly human CYPs are now available, as well as other xenobiotic-metabolizing enzymes such as DT-diaphorase and glutathione $S$-transferases. The crystallization and X-ray structural elucidation of mammalian cytochromes $\mathrm{P} 450$ $[11,12]$ is a breakthrough that removes the set of assumptions inherent in homology modeling and will thus improve the predictive power of molecular modeling.

Quantum mechanical methods are also classified as local in Table 2. Here, a word of caution is necessary, since such methods are in principle applicable to any chemical system. However, they cannot handle more than one metabolic reaction or catalytic mechanism at a time, and as such can only predict metabolism in simple biological systems, in contrast to the global methods presented in the next subsection. 


\section{"Global” systems}

As reviewed by Hawkins [13], one approach to predict metabolism is to use databases in the form of either knowledge-based systems or predictive expert systems. Existing knowledge-based systems [14] include the MDL Metabolite Database [15], and the book series Biotransformations [16], which has been produced as a software product called Metabolism [17]. These databases can be searched to retrieve information on the known metabolism of compounds with similar structures or containing specific moieties.

Predictive databases attempt to portray the metabolites of a compound based on knowledge rules, defining the most likely products. Existing systems of this type are MetabolExpert [18], META [19], and METEOR [20].

\section{METEOR: A GLOBAL SYSTEM TO PREDICT METABOLISM}

\section{A brief presentation of METEOR}

METEOR is a computer system which uses a knowledge base of structure-metabolism rules (biotransformations) to predict the metabolic fate of a query chemical structure [21]. METEOR's biotransformation rules are generic reaction descriptors rather than simple entries in a reaction database. The system uses a rich internal structure representation language [22]. The expression of specific functional group transformations can, therefore, be made context-sensitive.

Unconstrained analyses of query structures can give rise to a combinatorial explosion of results data. To overcome this problem, METEOR has an integrated reasoning engine, based on a system of non-numerical argumentation $[23,24]$, which uses a repository of higher-level reasoning rules. The reasoning model built into METEOR allows the system to evaluate the likelihood of a biotransformation taking place. METEOR is provided with a link to an external $\log P$ calculation program (Clog $P$ [25]). The likelihood of biotransformation can modified by the reasoning engine according to the general, global relationship between lipophilicity and drug metabolism [6]. The system can also make comparisons between potentially competing biotransformations [26]. The user of the system can choose to analyze queries at a number of available search levels. At high levels, only the more likely biotransformations are requested for display. At lower levels, the more putative metabolites are also selected for display.

Input into the system is by graphical means or by molfile import. Output from the system is also flexible. Results can be viewed from within the program where individual metabolites can be selected and processed to deeper levels or sent to DEREK for Windows [27] for a toxicological assessment. The metabolic tree can be searched by molecular mass, molecular formula, or structure. Reports can be generated in rich text format, and metabolites can be exported in SDfile format.

The system is also supplied with a knowledge base editor so that users can build their own biotransformations and rules. The predictive capability of the system is constantly being evaluated, and the knowledge base and rule base are continuously being developed and improved.

\section{An evaluation of METEOR}

We have recently completed an evaluation study of the now outdated version 6.0 of METEOR, comparing its predictions ("retrodictions" or "retrospective results" would be more appropriate here) with the published fate of nine drugs and one industrial chemical. Criteria for the selection of these 10 substrates were impeccable biological data available from the literature, the chemical diversity of the substrates, and extensive metabolism with multiple pathways. The 10 substrates were acenocoumarol, diclofenac, esonarimod, etoperidone, galantamine, gemifloxacine, $n$-hexane, indinavir, omapatrilat, and tramadol. 
The results for acenocoumarol are summarized in Figs. 1A and 1B. There was a full qualitative agreement between experiment and algorithm for seven reactions (Fig. 1A), five of which are clearly mediated by cytochromes P450. Three reactions were predicted, but not seen (false positives). Reactions $\mathbf{m A 8}$ and $\mathbf{m A 9}$ are reasonable ones which may have escaped experimental detection due to low levels. In contrast, hydrolytic ring opening of functionalized coumarins (mA10) is poorly documented if at all [4], suggesting that additional structural constraints need to be implemented in the software. Cytochrome P450-mediated reactions of dehydrogenation are rare, but greatly facilitated for highly delocalized metabolites [3], as is the case here (eA11). This false negative offers a valuable example of how an evaluation can help update the biotransformation dictionary of global systems.

For the 10 substrates, 130 first-generation metabolites were predicted and/or seen experimentally. Correct predictions represented $30 \%$ of these reactions, apparently false positives $62 \%$, and false negatives $8 \%$. These $70 \%$ discrepant results could be subdivided into $20 \%$ apparently false positives due to molecular constraints not being taken into account, $3 \%$ false negatives due to missing reactions in the biotransformation dictionary, and $47 \%$ most probably due to the complexity of biological factors. As stated, such results are of considerable interest in improving and updating the algorithm.

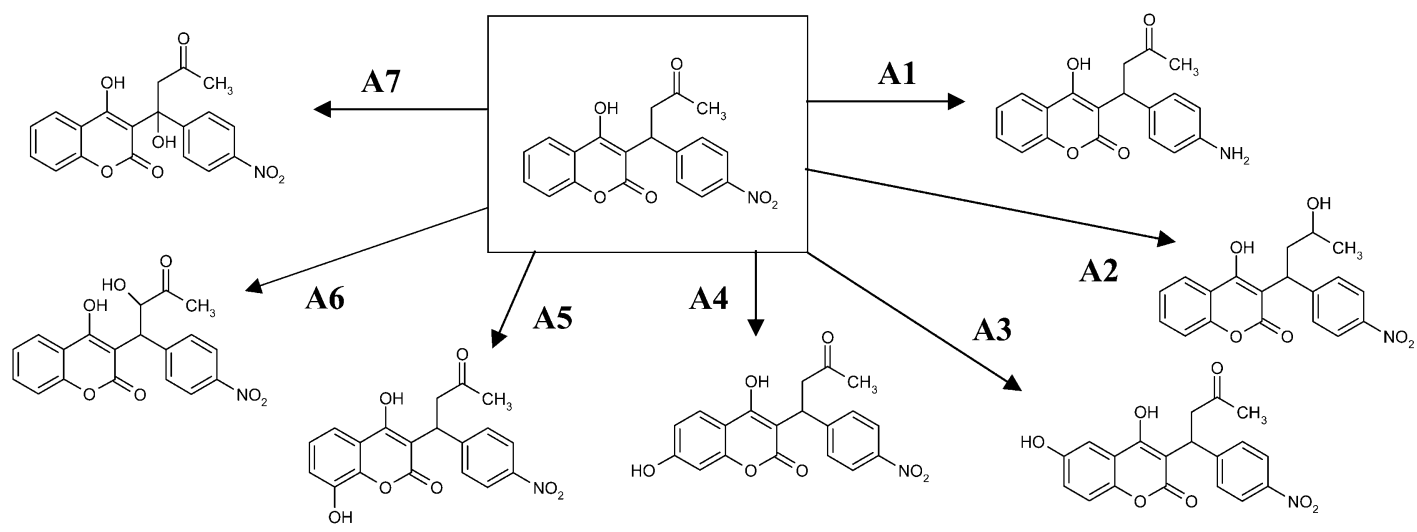

Seen experimentally

A1

A2

Nitro reduction

A3

Ketone reduction

A4

Phenol formation at C6

A5

Phenol formation at $\mathrm{C} 7$

A6

A7
Phenol formation at $\mathrm{C} 8$

C-Hydroxylation $\alpha$ to $\mathrm{C}=\mathrm{O}$

Benzylic hydroxylation
Predicted by METEOR

Plausible

Plausible

Plausible

Plausible

Plausible

Probable

Plausible

Fig. 1A Metabolic scheme of acenocoumarol, comparing the experimental results [28] with the predictions of METEOR version 6.0 [20]. (A) Metabolic reactions predicted and seen experimentally. (B) False positives (predicted but not seen experimentally) and false negative (seen but not predicted). 


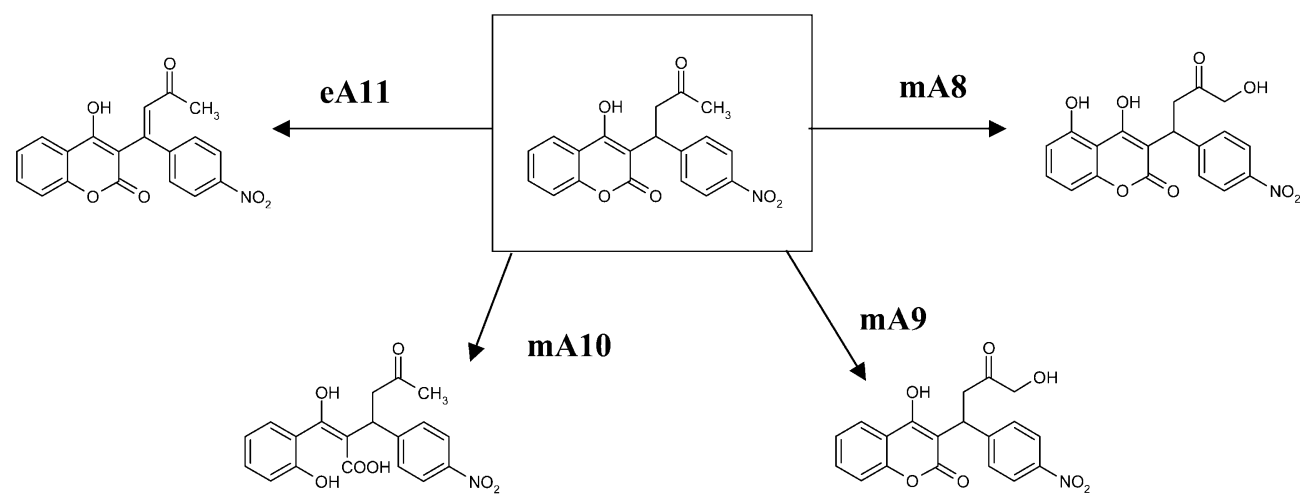

\section{Predicted by METEOR}

mA8 Plausible: Phenol formation at $\mathrm{C} 5$

mA9 Probable: Terminal hydroxylation alpha to $\mathrm{C}=\mathrm{O}$

mA10 Probable: Hydrolytic lactone opening

\section{Seen experimentally}

\section{eA11 Dehydrogenation}

Fig. 1B Metabolic scheme of acenocoumarol, comparing the experimental results [28] with the predictions of METEOR version 6.0 [20]. False positives (predicted but not seen experimentally) and false negative (seen but not predicted).

\section{CONCLUSION}

Drug metabolism, like any other biological response, is heavily dependent on the molecular properties of substrates. And this is an encouraging statement, since it implies that structure-metabolism relationships do exist and hence that predictions are possible. Indeed, many local predictions have been reported. These usually allow useful predictions within the explored space of molecular diversity and property.

Global predictions are much more ambitious and, therefore, remain a greater challenge. Of course, current expert systems will offer qualitative predictions as to possible metabolites, but the danger of false positives and false negatives is ever present. This is due to the complexity of molecular structure, the diversity of modes of interactions between substrates and metabolizing systems, and, above all, the many biological factors involved. And if one general conclusion should emerge from all what biologists have learned about biological regulations, it is that biological factors are interdependent in a nonlinear manner. In other words, these factors not only influence the biological responses, they also influence each other. The implication is that the systematic study of biological factors taken one by one or few by few will never yield a complete understanding of biological regulations. 


\section{REFERENCES}

1. B. Testa, H. van de Waterbeemd, G. Folkers, R. Guy (Eds.). Pharmacokinetic Optimization in Drug Research: Biological, Physicochemical and Computational Strategies, Verlag Helvetica Chimica Acta, Zurich (2001).

2. B. Testa and W. Soine. In Burger's Medicinal Chemistry and Drug Discovery, D. J. Abraham (Ed.), $6^{\text {th }}$ ed., Vol. 2, pp. 431-498, Wiley-Interscience, Hoboken, NJ (2003).

3. B. Testa. The Metabolism of Drugs and Other Xenobiotics: Biochemistry of Redox Reactions, Academic Press, London (1995).

4. B. Testa and J. M. Mayer. Hydrolysis in Drug and Prodrug Metabolism: Chemistry, Biochemistry and Enzymology, Wiley-Verlag Helvetica Chimica Acta, Zurich (2003).

5. A. G. E. Wilson, A. C. White, R. A. Mueller. Curr. Opin. Drug Discov. Dev. 6, 123 (2003).

6. B. Testa, P. Crivori, M. Reist, P. A. Carrupt. Perspect. Drug Discov. Design 19, 179 (2000).

7. R. N. Hines, Z. Luo, T. Cresteil, X. Ding, R. A. Prough, J. L. Fitzpatrick, S. L. Ripp, K. C. Falkner, N. L. Ge, A. Levine, C. J. Elferink. Drug Metab. Disposit. 29, 623 (2001).

8. S. Ekins, D. M. Stresser, J. A. Williams. Trends Pharmacol. Sci. 24, 161 (2003).

9. A. M. ter Laak and N. P. E. Vermeulen. In Pharmacokinetic Optimization in Drug Research: Biological, Physicochemical and Computational Strategies, B. Testa, H. van de Waterbeemd, G. Folkers, R. Guy (Eds.), pp. 551-588, Verlag Helvetica Chimica Acta, Zurich (2001).

10. I. Zamora, L. Afzelius, G. Cruciani. J. Med. Chem. 46, 2313 (2003). See also <www.moldiscovery.com>.

11. P. A. Williams, J. Cosme, V. Sridhar, E. F. Johnson, D. E. McRee. Mol. Cell 5, 121 (2000).

12. P. A. Williams, J. Cosme, A. Ward, H. C. Angova, D. M. Vinkovic, H. Jhoti. Nature 424, 464 (2003)

13. D. R. Hawkins. Drug Discov. Today 4, 466 (1999).

14. P. W. Erhardt (Ed.). Metabolism Databases and High-Throughput Testing during Drug Design and Development, Blackwell Science, London (1999).

15. <www.mdl.com>

16. D. R. Hawkins (Ed.). Biotransformations: A Survey of the Biotransformations of Drugs and Chemicals in Animals, Vols. 1-7, Royal Society of Chemistry, London (1988-1996).

17. <www.accelrys.com>

18. <www.compudrug.com>

19. <www.multicase.com>

20. $<$ www.lhasalimited.org $>$

21. J. J. Langowski and A. Long. Adv. Drug Deliv. Rev. 54, 407 (2002).

22. C. A. G. Tonnelier, J. Fox, P. N. Judson, P. J. Krause, N. Pappas, M. Patel. J. Chem. Inf. Comp. Sci. 37, 117 (1997).

23. P. N. Judson, J. Fox, P. J. Krause. J. Chem. Inf. Comp. Sci. 36, 621 (1996).

24. P. N. Judson and J. D. Vessey. J. Chem. Inf. Comp. Sci. 43, 1356 (2003).

25. <www.biobyte.com>

26. W. G. Button, P. N. Judson, A. Long, J. D. Vessey. J. Chem. Inf. Comp. Sci. 43, 1371 (2003).

27. P. N. Judson, C. A. Marchant, J. D. Vessey. J. Chem. Inf. Comp. Sci. 43, 1364 (2003).

28. J. J. Hermans and H. H. W. Thijssen. Xenobiotica 21, 295 (1991). 